\title{
A Hájek-Rényi-Type Maximal Inequality and Strong Laws of Large Numbers for Multidimensional Arrays
}

\author{
Nguyen Van Quang' and Nguyen Van Huan² \\ ${ }^{1}$ Department of Mathematics, Vinh University, Nghe An 42000, Vietnam \\ ${ }^{2}$ Department of Mathematics, Dong Thap University, Dong Thap 871000, Vietnam \\ Correspondence should be addressed to Nguyen Van Huan, vanhuandhdt@yahoo.com
}

Received 1 July 2010; Accepted 27 October 2010

Academic Editor: Alexander I. Domoshnitsky

Copyright (C) 2010 N. V. Quang and N. Van Huan. This is an open access article distributed under the Creative Commons Attribution License, which permits unrestricted use, distribution, and reproduction in any medium, provided the original work is properly cited.

A Hájek-Rényi-type maximal inequality is established for multidimensional arrays of random elements. Using this result, we establish some strong laws of large numbers for multidimensional arrays. We also provide some characterizations of Banach spaces.

\section{Introduction and Preliminaries}

Throughout this paper, the symbol $C$ will denote a generic positive constant which is not necessarily the same one in each appearance. Let $d$ be a positive integer, the set of all nonnegative integer $d$-dimensional lattice points will be denoted by $\mathbb{N}_{0}^{d}$, and the set of all positive integer $d$-dimensional lattice points will be denoted by $\mathbb{N}^{d}$. We will write $\mathbf{1}, \mathbf{m}, \mathbf{n}$, and $\mathbf{n}+\mathbf{1}$ for points $(1,1, \ldots, 1),\left(m_{1}, m_{2}, \ldots, m_{d}\right),\left(n_{1}, n_{2}, \ldots, n_{d}\right)$, and $\left(n_{1}+1, n_{2}+1, \ldots, n_{d}+1\right)$, respectively. The notation $\mathbf{m} \leq \mathbf{n}$ (or $\mathbf{n} \geq \mathbf{m}$ ) means that $m_{i} \leqslant n_{i}$ for all $i=1,2, \ldots, d$, the limit $\mathbf{n} \rightarrow \infty$ is interpreted as $n_{i} \rightarrow \infty$ for all $i=1,2, \ldots, d$ (this limit is equivalent to $\left.\min \left\{n_{1}, n_{2}, \ldots, n_{d}\right\} \rightarrow \infty\right)$, and we define $|\mathbf{n}|=\prod_{i=1}^{d} n_{i}$.

Let $\left\{b_{\mathbf{n}}, \mathbf{n} \in \mathbb{N}^{d}\right\}$ be a $d$-dimensional array of real numbers. We define $\Delta b_{\mathbf{n}}$ to be the $d$ th-order finite difference of the $b^{\prime}$ s at the point $\mathbf{n}$. Thus, $b_{\mathbf{n}}=\sum_{\mathbf{1} \leq \mathbf{k} \leq \mathbf{n}} \Delta b_{\mathbf{k}}$ for all $\mathbf{n} \in \mathbb{N}^{d}$. For example, if $d=2$, then for all $(i, j) \in \mathbb{N}^{2}, \Delta b_{i j}=b_{i j}-b_{i, j-1}-b_{i-1, j}+b_{i-1, j-1}$ (with the convention that $\left.b_{0,0}=b_{i, 0}=b_{0, j}=0\right)$. We say that $\left\{b_{\mathbf{n}}, \mathbf{n} \in \mathbb{N}^{d}\right\}$ is a nondecreasing array if $b_{\mathbf{k}} \leqslant b_{1}$ for any points $\mathbf{k} \preceq \mathbf{1}$.

Hájek and Rényi [1] proved the following important inequality: If $\left(X_{j}, j \geqslant 1\right)$ is a sequence of (real-valued) independent random variables with zero means and finite second 
moments, and $\left(b_{j}, j \geqslant 1\right)$ is a nondecreasing sequence of positive real numbers, then for any $\varepsilon>0$ and for any positive integers $n, n_{0}\left(n_{0}<n\right)$,

$$
\mathbb{P}\left(\max _{n_{0} \leqslant i \leqslant n} \frac{1}{b_{i}}\left|\sum_{j=1}^{i} X_{j}\right| \geqslant \varepsilon\right) \leqslant \frac{1}{\varepsilon^{2}}\left(\sum_{j=1}^{n_{0}} \frac{\mathbb{E} X_{j}^{2}}{b_{n_{0}}^{2}}+\sum_{j=n_{0}+1}^{n} \frac{\mathbb{E} X_{j}^{2}}{b_{j}^{2}}\right) .
$$

This inequality is a generalization of the Kolmogorov inequality and is a useful tool to prove the strong law of large numbers. Fazekas and Klesov [2] gave a general method for obtaining the strong law of large numbers for sequences of random variables by using a Hájek-Rényi-type maximal inequality. Afterwards, Noszály and Tómács [3] extended this result to multidimensional arrays (see also Klesov et al. [4]). They provided a sufficient condition for $d$-dimensional arrays of random variables to satisfy the strong law of large numbers

$$
\frac{1}{b_{\mathbf{n}}} \sum_{1 \leq \mathbf{k} \leq \mathbf{n}} X_{\mathbf{k}} \longrightarrow 0 \quad \text { a.s. as } \mathbf{n} \longrightarrow \infty
$$

where $\left\{b_{\mathbf{n}}, \mathbf{n} \in \mathbb{N}^{d}\right\}$ is a positive, nondecreasing $d$-sequence of product type, that is, $b_{\mathbf{n}}=$ $\prod_{i=1}^{d} b_{n_{i}}^{(i)}$, where $\left\{b_{n_{i}}^{(i)}, n_{i} \geqslant 1\right\}$ is a nondecreasing sequence of positive real numbers for each $i=1,2, \ldots, d$. Then, we have

$$
b_{\mathbf{n}}=\sum_{1 \leq \mathbf{k} \leq \mathbf{n}} \Delta b_{\mathbf{k}}=b_{n_{1}}^{(1)} b_{n_{2}}^{(2)} \cdots b_{n_{d}}^{(d)}, \quad \mathbf{n} \in \mathbb{N}^{d} .
$$

This implies that

$$
\Delta b_{\mathbf{n}}=\left(b_{n_{1}}^{(1)}-b_{n_{1}-1}^{(1)}\right)\left(b_{n_{2}}^{(2)}-b_{n_{2}-1}^{(2)}\right) \cdots\left(b_{n_{d}}^{(d)}-b_{n_{d}-1}^{(d)}\right), \quad \mathbf{n} \in \mathbb{N}^{d} .
$$

Therefore,

$$
\begin{gathered}
\Delta b_{\mathbf{n}} \geqslant 0, \quad \mathbf{n} \in \mathbb{N}^{d}, \\
\Delta b_{\mathbf{n}} \Delta b_{\mathbf{n}+1}=\Delta b_{n_{1} n_{2} \cdots n_{d-1}, n_{d}+1} \Delta b_{n_{1}+1, n_{2}+1, \ldots, n_{d-1}+1, n_{d}}, \quad \mathbf{n} \in \mathbb{N}^{d} .
\end{gathered}
$$

On the other hand, we can show that under the assumption that $\left\{b_{\mathbf{n}}, \mathbf{n} \in \mathbb{N}^{d}\right\}$ is an array of positive real numbers satisfying (1.5), it is not possible to guarantee that (1.6) holds (for details, see Example 2.8 in the next section).

Thus, if $\left\{b_{\mathbf{n}}, \mathbf{n} \in \mathbb{N}^{d}\right\}$ is a positive, nondecreasing $d$-sequence of product type, then it is an array of positive real numbers satisfying (1.5), but the reverse is not true.

In this paper, we use the hypothesis that $\left\{b_{\mathbf{n}}, \mathbf{n} \in \mathbb{N}^{d}\right\}$ is an array of positive real numbers satisfying (1.5) and continue to study the problem of finding the sufficient condition for the strong law of large numbers (1.2). We also establish a Hájek-Rényi-type maximal inequality for multidimensional arrays of random elements and some maximal moment inequalities for arrays of dependent random elements. 
The paper is organized as follows. In the rest of this section, we recall some definitions and present some lemmas. Section 2 is devoted to our main results and their proofs.

Let $(\Omega, \mathcal{F}, \mathbb{P})$ be a probability space. A family $\left\{\mathcal{F}_{\mathbf{n}}, \mathbf{n} \in \mathbb{N}_{0}^{d}\right\}$ of nondecreasing sub- $\sigma$ algebras of $\mathcal{F}$ related to the partial order $\leq$ on $\mathbb{N}_{0}^{d}$ is said to be a stochastic basic.

Let $\left\{\mathcal{F}_{\mathbf{n}}, \mathbf{n} \in \mathbb{N}_{0}^{d}\right\}$ be a stochastic basic such that $\mathcal{F}_{\mathbf{n}}=\{\emptyset, \Omega\}$ if $|\mathbf{n}|=0$, let $\mathbf{E}$ be a real separable Banach space, let $\boldsymbol{B}(\mathbf{E})$ be the $\sigma$-algebra of all Borel sets in $\mathbf{E}$, and let $\left\{X_{\mathbf{n}}, \mathbf{n} \in \mathbb{N}^{d}\right\}$ be an array of random elements such that $X_{\mathbf{n}}$ is $\mathscr{F}_{\mathbf{n}} / \mathbb{B}(\mathbf{E})$-measurable for all $\mathbf{n} \in \mathbb{N}^{d}$. Then $\left\{X_{\mathbf{n}}, \mathcal{F}_{\mathbf{n}}, \mathbf{n} \in \mathbb{N}^{d}\right\}$ is said to be an adapted array.

For a given stochastic basic $\left\{\mathcal{F}_{\mathbf{n}}, \mathbf{n} \in \mathbb{N}_{0}^{d}\right\}$, for $\mathbf{n} \in \mathbb{N}_{0}^{d}$, we set

$$
\begin{gathered}
\mathcal{F}_{\mathbf{n}}^{1}=\bigvee_{k_{i} \geqslant 1} \mathcal{F}_{(2 \leqslant i \leqslant d)} \boldsymbol{F}_{n_{1} k_{2} k_{3} \cdots k_{d}}:=\bigvee_{k_{2}=1}^{\infty} \bigvee_{k_{3}=1}^{\infty} \cdots \bigvee_{k_{d}=1}^{\infty} \mathcal{F}_{n_{1} k_{2} k_{3} \cdots k_{d} \prime} \\
\mathcal{F}_{\mathbf{n}}^{j}=\bigvee_{k_{i} \geqslant 1} \bigvee_{(1 \leqslant i \leqslant j-1)} \mathcal{F}_{k_{i} \geqslant 1 \cdots k_{j-1} n_{j} k_{j+1} \cdots k_{d}} \text { if } 1<j<d, \\
\mathcal{F}_{\mathbf{n}}^{d}=\bigvee_{\left.k_{i} \geqslant 1+1 \leqslant i \leqslant d\right)} \bigvee_{(1 \leqslant i \leqslant d-1)} \mathcal{F}_{k_{1} k_{2} \cdots k_{d-1} n_{d} \prime}
\end{gathered}
$$

in the case $d=1$, we set $\mathcal{F}_{n}^{1}=\mathcal{F}_{n}$.

An adapted array $\left\{X_{\mathbf{n}}, \mathcal{F}_{\mathbf{n}}, \mathbf{n} \in \mathbb{N}^{d}\right\}$ is said to be a martingale difference array if $\mathbb{E}\left(X_{\mathbf{n}} \mid \mathcal{F}_{\mathbf{n}-1}^{i}\right)=0$ for all $\mathbf{n} \in \mathbb{N}^{d}$ and for all $i=1,2, \ldots, d$.

In Quang and Huan [5], the authors showed that the set of all martingale difference arrays is really larger than the set of all arrays of independent mean zero random elements.

A Banach space $\mathbf{E}$ is said to be $p$-uniformly smooth $(1 \leqslant p \leqslant 2)$ if

$$
\rho(\tau)=\sup \left\{\frac{\|x+y\|+\|x-y\|}{2}-1, \forall x, y \in \mathbf{E},\|x\|=1,\|y\|=\tau\right\}=\mathcal{O}\left(\tau^{p}\right) .
$$

A Banach space $\mathbf{E}$ is said to be $p$-smoothable if there exists an equivalent norm under which $\mathbf{E}$ is $p$-uniformly smooth.

Pisier [6] proved that a real separable Banach space $\mathbf{E}$ is $p$-smoothable $(1 \leqslant p \leqslant 2)$ if and only if there exists a positive constant $C$ such that for every $L^{p}$ integrable (E-valued) martingale difference sequence $\left\{X_{j}, 1 \leqslant j \leqslant n\right\}$,

$$
\mathbb{E}\left\|\sum_{j=1}^{n} X_{j}\right\|^{p} \leqslant C \mathbb{E} \sum_{j=1}^{n}\left\|X_{j}\right\|^{p} .
$$

In Quang and Huan [5], this inequality was used to define p-uniformly smooth Banach spaces.

Let $\left\{Y_{j}, j \geqslant 1\right\}$ be a sequence of independent identically distributed random variables with $\mathbb{P}\left(Y_{1}=1\right)=\mathbb{P}\left(Y_{1}=-1\right)=1 / 2$. Let $\mathbf{E}^{\infty}=\mathbf{E} \times \mathbf{E} \times \mathbf{E} \times \cdots$ and define

$$
\mathfrak{C}(\mathbf{E})=\left\{\left(v_{1}, v_{2}, \ldots\right) \in \mathbf{E}^{\infty}: \sum_{j=1}^{\infty} Y_{j} v_{j} \text { converges inprobability }\right\} .
$$


Let $1 \leqslant p \leqslant 2$. Then, $\mathrm{E}$ is said to be of Rademacher type $p$ if there exists a positive constant $C$ such that

$$
\mathbb{E}\left\|\sum_{j=1}^{\infty} \Upsilon_{j} v_{j}\right\|^{p} \leqslant C \sum_{j=1}^{\infty}\left\|v_{j}\right\|^{p} \quad \forall\left(v_{1}, v_{2}, \ldots\right) \in \mathfrak{C}(\mathbf{E}) .
$$

It is well known that if a real separable Banach space is of Rademacher type $p(1 \leqslant p \leqslant$ $2)$, then it is of Rademacher type $q$ for all $1 \leqslant q \leqslant p$. Every real separable Banach space is of Rademacher type 1 , while the $\mathcal{L}^{p}$-spaces and $\ell^{p}$-spaces are of Rademacher type $2 \wedge p$ for $p \geqslant 1$. The real line $\mathbb{R}$ is of Rademacher type 2 . Furthermore, if a Banach space is $p$-smoothable, then it is of Rademacher type $p$. For more details, the reader may refer to Borovskikh and Korolyuk [7], Pisier [8], and Woyczyński [9].

Now, we present some lemmas which will be needed in what follows. The first lemma is a variation of Lemma 2.6 of Fazekas and Tómács [10] and is a multidimensional version of the Kronecker lemma.

Lemma 1.1. Let $\left\{x_{\mathbf{n}}, \mathbf{n} \in \mathbb{N}^{d}\right\}$ be an array of nonnegative real numbers, and let $\left\{b_{\mathbf{n}}, \mathbf{n} \in \mathbb{N}^{d}\right\}$ be a nondecreasing array of positive real numbers such that $b_{\mathbf{n}} \rightarrow \infty$ as $\mathbf{n} \rightarrow \infty$. If

$$
\sum_{\mathbf{n} \geq 1} x_{\mathbf{n}}<\infty
$$

then

$$
\frac{1}{b_{\mathbf{n}}} \sum_{1 \leq \mathbf{k} \leq \mathbf{n}} b_{\mathbf{k}} x_{\mathbf{k}} \longrightarrow 0 \quad \text { as } \mathbf{n} \longrightarrow \infty .
$$

Proof. For every $\varepsilon>0$, there exists a point $\mathbf{n}_{0} \in \mathbb{N}^{d}$ such that

$$
\sum_{\mathbf{k} \geq 1} x_{\mathbf{k}}-\sum_{\mathbf{1} \leq \mathbf{k} \leq \mathbf{n}_{0}} x_{\mathbf{k}} \leqslant \varepsilon
$$

Therefore, for all $\mathbf{n} \geq \mathbf{n}_{0}$,

$$
0 \leqslant \frac{1}{b_{\mathbf{n}}}\left(\sum_{1 \leq \mathbf{k} \leq \mathrm{n}} b_{\mathbf{k}} x_{\mathrm{k}}-\sum_{1 \leq \mathbf{k} \leq \mathrm{n}_{0}} b_{\mathrm{k}} x_{\mathrm{k}}\right) \leqslant\left(\sum_{1 \leq \mathbf{k} \leq \mathrm{n}} x_{\mathrm{k}}-\sum_{1 \leq \mathbf{k} \leq \mathrm{n}_{0}} x_{\mathrm{k}}\right) \leqslant \varepsilon .
$$

It means that

$$
\lim _{\mathbf{n} \rightarrow \infty} \frac{1}{b_{\mathbf{n}}}\left(\sum_{1 \leq \mathbf{k} \leq \mathbf{n}} b_{\mathbf{k}} x_{\mathbf{k}}-\sum_{1 \leq \mathbf{k} \leq \mathbf{n}_{0}} b_{\mathbf{k}} x_{\mathbf{k}}\right)=0 .
$$


On the other hand, since $b_{\mathbf{n}} \rightarrow \infty$ as $\mathbf{n} \rightarrow \infty$,

$$
\lim _{\mathbf{n} \rightarrow \infty} \frac{1}{b_{\mathbf{n}}} \sum_{1 \leq \mathbf{k} \leq \mathbf{n}_{0}} b_{\mathbf{k}} x_{\mathbf{k}}=0
$$

Combining the above arguments, this completes the proof of Lemma 1.1.

The proof of the next lemma is very simple and is therefore omitted.

Lemma 1.2. Let $(\Omega, \mathcal{F}, \mathbb{P})$ be a probability space, and let $\left\{A_{\mathbf{n}}, \mathbf{n} \in \mathbb{N}^{d}\right\}$ be an array of sets in $\mathcal{F}$ such that $A_{\mathbf{n}} \subset A_{\mathrm{m}}$ for any points $\mathbf{m} \leq \mathbf{n}$. Then,

$$
\mathbb{P}\left(\bigcap_{n \geq 1} A_{\mathrm{n}}\right)=\lim _{\mathrm{n} \rightarrow \infty} \mathbb{P}\left(A_{\mathrm{n}}\right) .
$$

Lemma 1.3. Let $\left\{X_{\mathbf{n}}, \mathbf{n} \in \mathbb{N}^{d}\right\}$ be an array of random elements. If for any $\varepsilon>0$,

$$
\lim _{\mathbf{n} \rightarrow \infty} \mathbb{P}\left(\sup _{\mathbf{k} \geq \mathbf{n}}\left\|X_{\mathbf{k}}\right\| \geqslant \varepsilon\right)=0,
$$

then $X_{\mathbf{n}} \rightarrow 0$ a.s. as $\mathbf{n} \rightarrow \infty$.

Proof. For each $i \geqslant 1$, we have

$$
\begin{aligned}
\mathbb{P}\left(\bigcap_{\mathbf{n} \geq 1} \bigcup_{\mathbf{k} \geq \mathbf{n}}\left(\left\|X_{\mathbf{k}}\right\| \geqslant \frac{1}{i}\right)\right) & =\lim _{\mathbf{n} \rightarrow \infty} \mathbb{P}\left(\bigcup_{\mathbf{k} \geq \mathbf{n}}\left(\left\|X_{\mathbf{k}}\right\| \geqslant \frac{1}{i}\right)\right) \quad \text { (by Lemma 1.2) } \\
& \leqslant \lim _{\mathbf{n} \rightarrow \infty} \mathbb{P}\left(\sup _{\mathbf{k} \geq \mathbf{n}}\left\|X_{\mathbf{k}}\right\| \geqslant \frac{1}{i}\right)=0 .
\end{aligned}
$$

Set

$$
A=\bigcup_{i \geqslant 1} \bigcap_{\mathbf{n} \geq 1}\left(\left\|X_{\mathbf{k} \geq \mathbf{n}}\right\| \geqslant \frac{1}{i}\right)
$$

Then, $\mathbb{P}(A)=0$ and for all $\omega \notin A$, for any $i \geqslant 1$, there exists a point $\mathbf{l} \in \mathbb{N}^{d}$ such that $\left\|X_{\mathbf{k}}(\omega)\right\|<$ $1 / i$ for all $\mathbf{k} \geq 1$. It means that

$$
X_{\mathbf{k}} \longrightarrow 0 \quad \text { a.s. as } k \longrightarrow \infty \text {. }
$$

The proof is completed.

Lemma 1.4 (Quang and Huan [5]). Let $1 \leqslant p \leqslant 2$, and let $\mathbf{E}$ be a real separable Banach space. Then, the following two statements are equivalent. 
(i) The Banach space $\mathbf{E}$ is p-smoothable.

(ii) For every $L^{p}$ integrable martingale difference array $\left\{X_{\mathbf{n}}, \boldsymbol{F}_{\mathbf{n}}, \mathbf{n} \in \mathbb{N}^{d}\right\}$, there exists a positive constant $C_{(p, d)}$ (depending only on $p$ and $d$ ) such that

$$
\mathbb{E}\left\|\sum_{1 \leq \mathbf{k} \leq \mathbf{n}} X_{\mathbf{k}}\right\|^{p} \leqslant C_{(p, d)} \sum_{1 \leq \mathbf{k} \leq \mathbf{n}} \mathbb{E}\left\|X_{\mathbf{k}}\right\|^{p}, \quad \mathbf{n} \in \mathbb{N}^{d} .
$$

\section{Main Results}

Theorem 2.1 provides a Hájek-Rényi-type maximal inequality for multidimensional arrays of random elements. This theorem is inspired by the work of Shorack and Smythe [11].

Theorem 2.1. Let $p>0$, let $\left\{b_{\mathbf{n}}, \mathbf{n} \in \mathbb{N}^{d}\right\}$ be an array of positive real numbers satisfying (1.5), and let $\left\{X_{\mathbf{n}}, \mathbf{n} \in \mathbb{N}^{d}\right\}$ be an array of random elements in a real separable Banach space. Then, there exists a positive constant $C_{(p, d)}$ such that for any $\varepsilon>0$ and for any points $\mathbf{m} \preceq \mathbf{n}$,

$$
\mathbb{P}\left(\max _{\mathbf{m} \leq \mathbf{k} \leq \mathbf{n}} \frac{1}{b_{\mathbf{k}}}\left\|\sum_{1 \leq 1 \leq \mathbf{k}} X_{1}\right\| \geqslant \varepsilon\right) \leqslant \frac{C_{(p, d)}}{\varepsilon^{p}} \mathbb{E} \max _{1 \leq \mathbf{k} \leq \mathbf{n}}\left\|\sum_{1 \leq 1 \leq \mathbf{k}} \frac{X_{1}}{b_{1}+b_{\mathbf{m}}}\right\|^{p} .
$$

Proof. Since $\left\{b_{\mathbf{n}}, \mathbf{n} \in \mathbb{N}^{d}\right\}$ is a nondecreasing array of positive real numbers,

$$
\begin{aligned}
\mathbb{P}\left(\max _{\mathbf{m} \leq \mathbf{k} \leq \mathbf{n}} \frac{1}{b_{\mathbf{k}}}\left\|\sum_{1 \leq 1 \leq \mathbf{k}} X_{1}\right\| \geqslant \varepsilon\right) & \leqslant \mathbb{P}\left(\max _{\mathbf{m} \leq \mathbf{k} \leq \mathbf{n}} \frac{1}{b_{\mathbf{k}}+b_{\mathbf{m}}}\left\|\sum_{1 \leq 1 \leq \mathbf{k}} X_{1}\right\| \geqslant \frac{\varepsilon}{2}\right) \\
& \leqslant \mathbb{P}\left(\max _{1 \leq \mathbf{k} \leq \mathbf{n}} \frac{1}{b_{\mathbf{k}}+b_{\mathbf{m}}}\left\|\sum_{1 \leq 1 \leq \mathbf{k}} X_{1}\right\| \geqslant \frac{\varepsilon}{2}\right) .
\end{aligned}
$$

For $\mathbf{k} \in \mathbb{N}^{d}$, set

$$
r_{\mathrm{k}}=b_{\mathrm{k}}+b_{\mathrm{m}}, \quad D_{\mathrm{k}}=\sum_{1 \leq \mathbf{l} \leq \mathrm{k}} \frac{X_{1}}{r_{1}}
$$

Then, by interchanging the order of summation, we obtain the following

$$
\sum_{1 \leq 1 \leq \mathrm{k}} X_{1}=\sum_{1 \leq 1 \leq \mathrm{k}}\left(\sum_{1 \leq t \leq 1} \Delta r_{\mathrm{t}}\right) \frac{X_{1}}{r_{1}}=\sum_{1 \leq \mathrm{t} \leq \mathrm{k}} \Delta r_{\mathrm{t}}\left(\sum_{\mathrm{t} \leq 1 \leq \mathrm{k}} \frac{X_{1}}{r_{1}}\right) .
$$

Thus, since $\Delta r_{\mathrm{t}} \geqslant 0$,

$$
\max _{1 \leq \mathbf{k} \leq \mathrm{n}} \frac{1}{r_{\mathbf{k}}}\left\|\sum_{1 \leq 1 \leq \mathbf{k}} X_{1}\right\| \leqslant 2^{d} \max _{1 \leq 1 \leq \mathrm{n}}\left\|D_{1}\right\|
$$


By (2.2) and (2.5) and the Markov inequality, we have

$$
\begin{aligned}
\mathbb{P}\left(\max _{\mathbf{m} \leq \mathbf{k} \leq \mathbf{n}} \frac{1}{b_{\mathbf{k}}}\left\|\sum_{1 \leq 1 \leq \mathbf{k}} X_{1}\right\| \geqslant \varepsilon\right) & \leqslant \mathbb{P}\left(\max _{1 \leq 1 \leq \mathbf{n}}\left\|D_{1}\right\| \geqslant \frac{\varepsilon}{2^{d+1}}\right) \\
& \leqslant \frac{2^{p(d+1)}}{\varepsilon^{p}} \mathbb{E} \max _{1 \leq \mathbf{l} \mathbf{n}}\left\|D_{1}\right\|^{p} .
\end{aligned}
$$

This completes the proof of the theorem.

Now, we use Theorem 2.1 to prove a strong law of large numbers for multidimensional arrays of random elements. This result is inspired by Theorem 3.2 of Klesov et al. [4].

Theorem 2.2. Let $p>0$, let $\left\{a_{\mathbf{n}}, \mathbf{n} \in \mathbb{N}^{d}\right\}$ be an array of nonnegative real numbers, let $\left\{b_{\mathbf{n}}, \mathbf{n} \in \mathbb{N}^{d}\right\}$ be an array of positive real numbers satisfying (1.5) and $b_{\mathbf{n}} \rightarrow \infty$ as $\mathbf{n} \rightarrow \infty$, and let $\left\{X_{\mathbf{n}}, \mathbf{n} \in \mathbb{N}^{d}\right\}$ be an array of random elements in a real separable Banach space such that for any points $\mathbf{m} \leq \mathbf{n}$,

$$
\mathbb{E} \max _{1 \leq \mathbf{k} \leq \mathbf{n}}\left\|\sum_{1 \leq 1 \leq \mathbf{k}} \frac{X_{1}}{b_{1}+b_{\mathrm{m}}}\right\|^{p} \leqslant C \sum_{1 \leq \mathbf{k} \leq \mathbf{n}} \frac{a_{\mathbf{k}}}{\left(b_{\mathbf{k}}+b_{\mathbf{m}}\right)^{p}} .
$$

Then, the condition

$$
\sum_{\mathbf{n} \geq 1} \frac{a_{\mathbf{n}}}{b_{\mathbf{n}}^{p}}<\infty
$$

implies (1.2).

Proof. By (2.7) and Theorem 2.1, for any $\varepsilon>0$ and for any points $\mathbf{m} \preceq \mathbf{n}$, we have

$$
\mathbb{P}\left(\max _{\mathbf{m} \leq \mathbf{k} \leq \mathbf{n}} \frac{1}{b_{\mathbf{k}}}\left\|\sum_{1 \leq 1 \leq \mathbf{k}} X_{1}\right\| \geqslant \varepsilon\right) \leqslant \frac{C}{\varepsilon^{p}} \sum_{1 \leq \mathbf{k} \leq \mathbf{n}} \frac{a_{\mathbf{k}}}{\left(b_{\mathbf{k}}+b_{\mathbf{m}}\right)^{p}} .
$$

This implies, by letting $\mathbf{n} \rightarrow \infty$, that

$$
\begin{aligned}
\mathbb{P}\left(\sup _{\mathbf{k} \geq \mathrm{m}} \frac{1}{b_{\mathrm{k}}}\left\|\sum_{1 \leq 1 \leq \mathbf{k}} X_{1}\right\| \geqslant \varepsilon\right) & \leqslant \frac{C}{\varepsilon^{p}} \sum_{\mathbf{k} \geq 1} \frac{a_{\mathbf{k}}}{\left(b_{\mathbf{k}}+b_{\mathrm{m}}\right)^{p}} \\
& \leqslant \frac{C}{\varepsilon^{p}}\left(\sum_{1 \leq \mathbf{k} \leq \mathrm{m}} \frac{a_{\mathbf{k}}}{b_{\mathrm{m}}^{p}}+\left(\sum_{\mathbf{k} \geq 1} \frac{a_{\mathbf{k}}}{b_{\mathbf{k}}^{p}}-\sum_{1 \leq \mathbf{k} \leq \mathrm{m}} \frac{a_{\mathbf{k}}}{b_{\mathbf{k}}^{p}}\right)\right) .
\end{aligned}
$$


Letting $\mathbf{m} \rightarrow \infty$, by (2.8) and Lemma 1.1, we obtain

$$
\lim _{\mathbf{m} \rightarrow \infty} \mathbb{P}\left(\sup _{\mathbf{k} \geq \mathbf{m}} \frac{1}{b_{\mathbf{k}}}\left\|\sum_{1 \leq \mathbf{l} \leq \mathbf{k}} X_{\mathbf{l}}\right\| \geqslant \varepsilon\right)=0 .
$$

Lemma 1.3 ensures that (1.2) holds. The proof is completed.

The next theorem provides three characterizations of $p$-smoothable Banach spaces. The equivalence of (i) and (ii) is an improvement of a result of Quang and Huan [5] (stated as Lemma 1.4 above).

Theorem 2.3. Let $1 \leqslant p \leqslant 2$, and let $\mathbf{E}$ be a real separable Banach space. Then, the following four statements are equivalent.

(i) The Banach space $\mathbf{E}$ is p-smoothable.

(ii) For every $L^{p}$ integrable martingale difference array $\left\{X_{\mathbf{n}}, \boldsymbol{F}_{\mathbf{n}}, \mathbf{n} \in \mathbb{N}^{d}\right\}$, there exists a positive constant $C_{(p, d)}$ such that

$$
\mathbb{E} \max _{1 \leq \mathbf{k} \leq \mathbf{n}}\left\|\sum_{1 \leq 1 \leq \mathbf{k}} X_{1}\right\|^{p} \leqslant C_{(p, d)} \sum_{1 \leq \mathbf{k} \leq \mathbf{n}} \mathbb{E}\left\|X_{\mathbf{k}}\right\|^{p}, \quad \mathbf{n} \in \mathbb{N}^{d} .
$$

(iii) For every $L^{p}$ integrable martingale difference array $\left\{X_{\mathbf{n}}, \boldsymbol{F}_{\mathbf{n}}, \mathbf{n} \in \mathbb{N}^{d}\right\}$, for every array of positive real numbers $\left\{b_{\mathbf{n}}, \mathbf{n} \in \mathbb{N}^{d}\right\}$ satisfying (1.5), for any $\varepsilon>0$, and for any points $\mathbf{m} \leq \mathbf{n}$, there exists a positive constant $C_{(p, d)}$ such that

$$
\mathbb{P}\left(\max _{\mathbf{m} \leq \mathbf{k} \leq \mathbf{n}} \frac{1}{b_{\mathbf{k}}}\left\|\sum_{1 \leq 1 \leq \mathbf{k}} X_{1}\right\| \geqslant \varepsilon\right) \leqslant \frac{C_{(p, d)}}{\varepsilon^{p}} \sum_{1 \leq \mathbf{k} \leq \mathbf{n}} \mathbb{E}\left\|\frac{X_{\mathbf{k}}}{b_{\mathbf{k}}+b_{\mathrm{m}}}\right\|^{p} .
$$

(iv) For every martingale difference array $\left\{X_{\mathbf{n}}, \boldsymbol{F}_{\mathbf{n}}, \mathbf{n} \in \mathbb{N}^{d}\right\}$, for every array of positive real numbers $\left\{b_{\mathbf{n}}, \mathbf{n} \in \mathbb{N}^{d}\right\}$ satisfying (1.5) and $b_{\mathbf{n}} \rightarrow \infty$ as $\mathbf{n} \rightarrow \infty$, the condition

$$
\sum_{\mathbf{n} \geq 1} \frac{\mathbb{E}\left\|X_{\mathbf{n}}\right\|^{p}}{b_{\mathbf{n}}^{p}}<\infty
$$

implies (1.2).

Proof. (i) $\Rightarrow$ (ii): We easily obtain (2.12) in the case $p=1$. Now, we consider the case $1<p \leqslant 2$. By virtue of Lemma 1.4, it suffices to show that

$$
\mathbb{E} \max _{1 \leq \mathbf{k} \leq \mathbf{n}}\left\|\sum_{1 \leq 1 \leq \mathbf{k}} X_{1}\right\|^{p} \leqslant\left(\frac{p}{p-1}\right)^{p d} \mathbb{E}\left\|\sum_{1 \leq \mathbf{k} \leq \mathbf{n}} X_{\mathbf{k}}\right\|^{p}, \quad \mathbf{n} \in \mathbb{N}^{d} .
$$

First, we remark that for $d=1,(2.15)$ follows from Doob's inequality. We assume that (2.15) holds for $d=D-1 \geqslant 1$, we wish to show that it holds for $d=D$. 
Journal of Inequalities and Applications

For $\mathbf{k} \in \mathbb{N}^{D}$, we set

$$
S_{\mathbf{k}}=\sum_{1 \leq 1 \leq \mathbf{k}} X_{\mathbf{l}}, \quad Y_{k_{D}}=\max _{1 \leqslant k_{i} \leqslant n_{i}(1 \leqslant i \leqslant D-1)}\left\|S_{\mathbf{k}}\right\| .
$$

Then,

$$
\begin{aligned}
& \mathbb{E}\left(S_{k_{1} k_{2} \cdots k_{D-1} k_{D}} \mid \boldsymbol{F}_{k_{1} k_{2} \cdots k_{D-1}, k_{D}-1}^{D}\right) \\
&= \mathbb{E}\left(S_{k_{1} k_{2} \cdots k_{D-1}, k_{D}-1} \mid \mathcal{F}_{k_{1} k_{2} \cdots k_{D-1}, k_{D}-1}^{D}\right) \\
&+\mathbb{E}\left(\sum_{1 \leqslant l_{i} \leqslant k_{i}(1 \leqslant i \leqslant D-1)} X_{l_{1} l_{2} \cdots l_{D-1} k_{D}} \mid \mathcal{F}_{k_{1} k_{2} \cdots k_{D-1}, k_{D}-1}^{D}\right) \\
&= S_{k_{1} k_{2} \cdots k_{D-1}, k_{D}-1} .
\end{aligned}
$$

Therefore,

$$
\begin{aligned}
\mathbb{E}\left(Y_{k_{D}} \mid \mathcal{F}_{k_{1} k_{2} \cdots k_{D-1}, k_{D}-1}^{D}\right) & =\mathbb{E}\left(\max _{1 \leqslant k_{i} \leqslant n_{i}(1 \leqslant i \leqslant D-1)}\left\|S_{\mathbf{k}}\right\| \mid \mathcal{F}_{k_{1} k_{2} \cdots k_{D-1}, k_{D}-1}^{D}\right) \\
& \geqslant \max _{1 \leqslant k_{i} \leqslant n_{i}(1 \leqslant i \leqslant D-1)}\left\|\mathbb{E}\left(S_{\mathbf{k}} \mid \boldsymbol{F}_{k_{1} k_{2} \cdots k_{D-1}, k_{D}-1}^{D}\right)\right\| \\
& =Y_{k_{D-1}} .
\end{aligned}
$$

It means that $\left\{Y_{k_{D}}, \Psi_{k_{1} k_{2} \cdots k_{D-1} k_{D}}^{D}, k_{D} \geqslant 1\right\}$ is a nonnegative submartingale. Applying Doob's inequality, we obtain

$$
\begin{aligned}
\mathbb{E} \max _{1 \leq \mathbf{k} \leq \mathbf{n}}\left\|S_{\mathbf{k}}\right\|^{p} & =\mathbb{E}\left(\max _{1 \leqslant k_{D} \leqslant n_{D}} Y_{k_{D}}\right)^{p} \leqslant\left(\frac{p}{p-1}\right)^{p} \mathbb{E} Y_{n_{D}}^{p} \\
& =\left(\frac{p}{p-1}\right)^{p} \underset{1 \leqslant k_{i} \leqslant n_{i}(1 \leqslant i \leqslant D-1)}{\mathbb{E}}\left\|S_{k_{1} k_{2} \cdots k_{D-1} n_{D}}\right\|^{p} .
\end{aligned}
$$

We set

$$
X_{k_{1} k_{2} \cdots k_{D-1}}^{(D-1)}=\sum_{k_{D}=1}^{n_{D}} X_{k_{1} k_{2} \cdots k_{D-1} k_{D}}, \quad \mathcal{F}_{k_{1} k_{2} \cdots k_{D-1}}^{(D-1)}=\bigvee_{k_{D}=1}^{\infty} \mathcal{F}_{k_{1} k_{2} \cdots k_{D-1} k_{D}}
$$


Then we again have that $\left\{X_{k_{1} k_{2} \cdots k_{D-1}}^{(D-1)}, \Psi_{k_{1} k_{2} \cdots k_{D-1}}^{(D-1)},\left(k_{1}, k_{2}, \ldots, k_{D-1}\right) \in \mathbb{N}^{D-1}\right\}$ is a martingale difference array. Therefore, by the inductive assumption, we obtain

$$
\begin{aligned}
& \mathbb{E} \max _{1 \leqslant k_{i} \leqslant n_{i}(1 \leqslant i \leqslant D-1)}\left\|S_{k_{1} k_{2} \cdots k_{D-1} n_{D}}\right\|^{p} \\
& =\mathbb{E}_{1 \leqslant k_{i} \leqslant n_{i}(1 \leqslant i \leqslant D-1)} \max _{1 \leqslant l_{i} \leqslant k_{i}(1 \leqslant i \leqslant D-1)} X_{l_{1} l_{2} \cdots l_{D-1}}^{(D-1)} \|^{p} \\
& \leqslant\left(\frac{p}{p-1}\right)^{p(D-1)} \mathbb{E}\left\|\sum_{1 \leqslant l_{i} \leqslant n_{i}(1 \leqslant i \leqslant D-1)} X_{l_{1} l_{2} \cdots l_{D-1}}^{(D-1)}\right\|^{p} \\
& =\left(\frac{p}{p-1}\right)^{p(D-1)} \mathbb{E}\left\|S_{n_{1} n_{2} \cdots n_{D}}\right\|^{p} .
\end{aligned}
$$

Combining (2.19) and (2.21) yields that (2.15) holds for $d=D$.

(ii) $\Rightarrow$ (iii): let $\left\{X_{\mathbf{n}}, \mathcal{F}_{\mathbf{n}}, \mathbf{n} \in \mathbb{N}^{d}\right\}$ be an arbitrary $L^{p}$ integrable martingale difference array. Then, for all $\mathbf{m} \in \mathbb{N}^{d},\left\{X_{\mathbf{n}} /\left(b_{\mathbf{n}}+b_{\mathbf{m}}\right), \boldsymbol{F}_{\mathbf{n}}, \mathbf{n} \in \mathbb{N}^{d}\right\}$ is also an $L^{p}$ integrable martingale difference array. Therefore, the assertion (ii) and Theorem 2.1 ensure that (2.13) holds.

(iii) $\Rightarrow$ (iv): the proof of this implication is similar to the proof of Theorem 2.2 and is therefore omitted.

(iv) $\Rightarrow$ (i): for a given positive integer $d$, assume that (iv) holds. Let $\left\{X_{j}, \mathcal{F}_{j}, j \geqslant 1\right\}$ be an arbitrary martingale difference sequence such that

$$
\sum_{j=1}^{\infty} \frac{\mathbb{E}\left\|X_{j}\right\|^{p}}{j^{p}}<\infty .
$$

For $\mathbf{n} \in \mathbb{N}^{d}$, set

$$
X_{\mathrm{n}}=X_{n_{1}} \text { if } n_{i}=1(2 \leqslant i \leqslant d),
$$

$X_{\mathbf{n}}=0 \quad$ if there exists a positive integer $i(2 \leqslant i \leqslant d)$ such that $n_{i}>1$,

$$
\mathcal{F}_{\mathrm{n}}=\mathscr{F}_{n_{1}}, \quad b_{\mathrm{n}}=n_{1} .
$$

Then, $\left\{X_{\mathbf{n}}, \mathcal{F}_{\mathbf{n}}, \mathbf{n} \in \mathbb{N}^{d}\right\}$ is a martingale difference array, and $\left\{b_{\mathbf{n}}, \mathbf{n} \in \mathbb{N}^{d}\right\}$ is an array of positive real numbers satisfying (1.5) and $b_{\mathbf{n}} \rightarrow \infty$ as $\mathbf{n} \rightarrow \infty$. Moreover, we see that

$$
\sum_{\mathbf{n} \geq 1} \frac{\mathbb{E}\left\|X_{\mathbf{n}}\right\|^{p}}{b_{\mathbf{n}}^{p}}=\sum_{n_{1}=1}^{\infty} \frac{\mathbb{E}\left\|X_{n_{1}}\right\|^{p}}{n_{1}^{p}}<\infty
$$


and so (1.2) holds. It means that

$$
\frac{1}{n_{1}} \sum_{j=1}^{n_{1}} X_{j} \longrightarrow 0 \quad \text { a.s. as } n_{1} \longrightarrow \infty
$$

Then, by Theorem 2.2 of Hoffmann-Jørgensen and Pisier [12], E is $p$-smoothable.

Remark 2.4. The inequality (2.15) holds for every $p>1$ and for every martingale difference array without imposing any geometric condition on the Banach space.

In the case $d=1$, Theorem 2.3 reduces to the following corollary which was proved by Gan [13] and Gan and Qiu [14].

Corollary 2.5. Let $1 \leqslant p \leqslant 2$, and let $\mathbf{E}$ be a real separable Banach space. Then, the following three statements are equivalent.

(i) The Banach space $\mathbf{E}$ is p-smoothable.

(ii) For every $L^{p}$ integrable martingale difference sequence $\left\{X_{j}, \boldsymbol{F}_{j}, j \geqslant 1\right\}$, for every nondecreasing sequence of positive real numbers $\left\{b_{j}, j \geqslant 1\right\}$, for any $\varepsilon>0$, and for any positive integers $n, n_{0}\left(n_{0}<n\right)$, there exists a positive constant $C$ such that

$$
\mathbb{P}\left(\max _{n_{0} \leqslant i \leqslant n} \frac{1}{b_{i}}\left\|\sum_{j=1}^{i} X_{j}\right\| \geqslant \varepsilon\right) \leqslant \frac{C}{\mathcal{E}^{p}}\left(\sum_{j=1}^{n_{0}} \frac{\mathbb{E}\left\|X_{j}\right\|^{p}}{b_{n_{0}}^{p}}+\sum_{j=n_{0}+1}^{n} \frac{\mathbb{E}\left\|X_{j}\right\|^{p}}{b_{j}^{p}}\right) .
$$

(iii) For every martingale difference sequence $\left\{X_{j}, \boldsymbol{F}_{j}, j \geqslant 1\right\}$ and for every nondecreasing sequence of positive real numbers $\left\{b_{j}, j \geqslant 1\right\}$ such that $b_{j} \rightarrow \infty$ as $j \rightarrow \infty$, the condition

$$
\sum_{j=1}^{\infty} \frac{\mathbb{E}\left\|X_{j}\right\|^{p}}{b_{j}^{p}}<\infty
$$

implies

$$
\frac{1}{b_{i}} \sum_{j=1}^{i} X_{j} \longrightarrow 0 \quad \text { a.s. as } i \longrightarrow \infty
$$

Remark 2.4 ensures that the inequality (2.15) holds for every $p>1$ and for every array of independent mean zero random elements in a real separable Banach space. Therefore, by using the implication $((2.1 .1) \Rightarrow(2.1 .2))$ of Theorem 2.1 of Hoffmann-Jørgensen and Pisier [12] and the same arguments as in the proof of Theorem 2.3, we get the following theorem which generalizes some results given by Christofides and Serfling [15] and Gan and Qiu [14]. We omit its proof.

Theorem 2.6. Let $1 \leqslant p \leqslant 2$, and let $\mathbf{E}$ be a real separable Banach space. Then, the following four statements are equivalent. 
(i) The Banach space $\mathbf{E}$ is of Rademacher type $p$.

(ii) For every array of $L^{p}$ integrable independent mean zero random elements $\left\{X_{\mathbf{n}}, \mathbf{n} \in \mathbb{N}^{d}\right\}$, there exists a positive constant $C_{(p, d)}$ such that (2.12) holds.

(iii) For every array of $L^{p}$ integrable independent mean zero random elements $\left\{X_{\mathbf{n}}, \mathbf{n} \in \mathbb{N}^{d}\right\}$, for every array of positive real numbers $\left\{b_{\mathbf{n}}, \mathbf{n} \in \mathbb{N}^{d}\right\}$ satisfying (1.5), for any $\varepsilon>0$, and for any points $\mathbf{m} \leq \mathbf{n}$, there exists a positive constant $C_{(p, d)}$ such that (2.13) holds.

(iv) For every array of independent mean zero random elements $\left\{X_{\mathbf{n}}, \mathbf{n} \in \mathbb{N}^{d}\right\}$, for every array of positive real numbers $\left\{b_{\mathbf{n}}, \mathbf{n} \in \mathbb{N}^{d}\right\}$ satisfying (1.5) and $b_{\mathbf{n}} \rightarrow \infty$ as $\mathbf{n} \rightarrow \infty$, the condition (2.14) implies (1.2).

We close this paper by giving a remark on Theorem 2.6 and an example which illustrates Theorems 2.2, 2.3, and 2.6.

Remark 2.7. By the same method as in the proof of Lemma 3 of Móricz et al. [16] and the same arguments as in the proof of Theorem 2.3, we can extend Theorem 2.6 to $\mathcal{M}$-dependent random fields.

Example 2.8. Let $d$ be a positive integer $(d \geqslant 2)$, and let $\left\{X_{\mathbf{n}}, \mathbf{n} \in \mathbb{N}^{d}\right\}$ be an array of independent random variables with

$$
\mathbb{P}\left(X_{\mathbf{n}}=-|\mathbf{n}|^{1 / 4}\right)=\mathbb{P}\left(X_{\mathbf{n}}=|\mathbf{n}|^{1 / 4}\right)=\frac{1}{2} .
$$

Then, $\left\{X_{\mathbf{n}}, \mathbf{n} \in \mathbb{N}^{d}\right\}$ is an array of independent mean zero random variables taking values in the 2-smoothable Banach space $\mathbb{R}$ (using the absolute value as norm).

$$
\begin{aligned}
& \text { Let } b_{\mathbf{n}}=|\mathbf{n}|+\min \left\{n_{1}, n_{2}, \ldots, n_{d}\right\}\left(\mathbf{n} \in \mathbb{N}^{d}\right) \text {. Then, } \\
& \qquad \Delta b_{\mathbf{n}}= \begin{cases}2 & \text { if } n_{1}=n_{2}=\cdots=n_{d}, \\
1 & \text { otherwise. }\end{cases}
\end{aligned}
$$

It means that $\left\{b_{\mathbf{n}}, \mathbf{n} \in \mathbb{N}^{d}\right\}$ is an array of positive real numbers satisfying (1.5) and $b_{\mathbf{n}} \rightarrow \infty$ as $\mathbf{n} \rightarrow \infty$. Moreover, by virtue of (1.6), we can show that $\left\{b_{\mathbf{n}}, \mathbf{n} \in \mathbb{N}^{d}\right\}$ is not a positive, nondecreasing $d$-sequence of product type. Therefore, (1.2) does not follow from Theorem 3.2 of Klesov et al. [4]. But for every array of positive real numbers $\left\{r_{\mathbf{n}}, \mathbf{n} \in \mathbb{N}^{d}\right\},\left\{X_{\mathbf{n}} / r_{\mathbf{n}}, \boldsymbol{F}_{\mathbf{n}}=\right.$ $\left.\sigma\left(X_{\mathbf{k}}, \mathbf{1} \preceq \mathbf{k} \preceq \mathbf{n}\right), \mathbf{n} \in \mathbb{N}^{d}\right\}$ is a martingale difference array such that

$$
\begin{gathered}
\mathbb{E} \max _{\mathbf{1} \leq \mathbf{k} \leq \mathbf{n}}\left|\sum_{1 \leq 1 \leq \mathbf{k}} \frac{X_{1}}{r_{1}}\right|^{2} \leqslant C \sum_{1 \leq \mathbf{k} \leq \mathbf{n}} \frac{\mathbb{E}\left|X_{\mathbf{k}}\right|^{2}}{r_{\mathbf{k}}^{2}}, \quad \mathbf{n} \in \mathbb{N}^{d} \quad(\text { by Theorem 2.3) } \\
\sum_{\mathbf{n} \geq 1} \frac{\mathbb{E}\left|X_{\mathbf{k}}\right|^{2}}{b_{\mathbf{n}}^{2}} \leqslant \sum_{\mathbf{n} \geq 1} \frac{|\mathbf{n}|^{1 / 2}}{|\mathbf{n}|^{2}}<\infty,
\end{gathered}
$$

and so (2.7) and (2.8) are satisfied, Theorem 2.2 ensures that (1.2) holds. 
As we know, the limit $|\mathbf{n}| \rightarrow \infty$ is equivalent to $\max \left\{n_{1}, n_{2}, \ldots, n_{d}\right\} \rightarrow \infty$. Recently, some authors have derived the sufficient conditions for the strong law of large numbers

$$
b_{\mathbf{n}}^{-1} \sum_{1 \leq \mathbf{k} \leq \mathbf{n}} X_{\mathbf{k}} \longrightarrow 0 \quad \text { a.s. as }|\mathbf{n}| \longrightarrow \infty,
$$

where $\left\{b_{\mathbf{n}}, \mathbf{n} \in \mathbb{N}^{d}\right\}$ is one of the special kinds of positive, nondecreasing $d$-sequences of product type. For more details, the reader may refer to [17-19]. Therefore, this example also shows that the implications ((i) $\Rightarrow$ (iv)) of Theorem 2.3 and ((i) $\Rightarrow$ (iv)) of Theorem 2.6 are independent of results obtained in [17-19].

\section{Acknowledgments}

The authors are grateful to the referee for carefully reading the paper and for offering some comments which helped to improve the paper. This research was supported by the National Foundation for Science Technology Development, Vietnam (NAFOSTED), no. 101.02.32.09.

\section{References}

[1] J. Hájek and A. Rényi, "Generalization of an inequality of Kolmogorov," Acta Mathematica Academiae Scientiarum Hungaricae, vol. 6, pp. 281-283, 1955.

[2] I. Fazekas and O. Klesov, "A general approach to the strong laws of large numbers," Theory of Probability and Its Applications, vol. 45, no. 3, pp. 436-449, 2002.

[3] C. Noszály and T. Tómács, "A general approach to strong laws of large numbers for fields of random variables," Annales Universitatis Scientiarum Budapestinensis de Rolando Eötvös Nominatae. Sectio Mathematica, vol. 43, pp. 61-78, 2001.

[4] O. Klesov, I. Fazekas, C. Noszály, and T. Tómács, "Strong laws of large numbers for sequences and fields," Theory of Stochastic Processes, vol. 5, no. 3-4, pp. 91-104, 2008.

[5] N. V. Quang and N. V. Huan, "A characterization of $p$-uniformly smooth Banach spaces and weak laws of large numbers for $d$-dimensional adapted arrays," The Indian Journal of Statistics, vol. 72, pp. 344-358, 2010.

[6] G. Pisier, "Martingales with values in uniformly convex spaces," Israel Journal of Mathematics, vol. 20, no. 3-4, pp. 326-350, 1975.

[7] Y. V. Borovskikh and V. S. Korolyuk, Martingale Approximation, VSP, Utrecht, The Netherlands, 1997.

[8] G. Pisier, "Probabilistic methods in the geometry of Banach spaces," in Probability and Analysis (Varenna, 1985), vol. 1206 of Lecture Notes in Mathematics, pp. 167-241, Springer, Berlin, Germany, 1986.

[9] W. A. Woyczyński, "On Marcinkiewicz-Zygmund laws of large numbers in Banach spaces and related rates of convergence," Probability and Mathematical Statistics, vol. 1, no. 2, pp. 117-131, 1981.

[10] I. Fazekas and T. Tómács, "Strong laws of large numbers for pairwise independent random variables with multidimensional indices," Publicationes Mathematicae Debrecen, vol. 53, no. 1-2, pp. 149-161, 1998.

[11] G. R. Shorack and R. T. Smythe, "Inequalities for $\left|S_{k}\right| / b_{k}$ where $k \in N^{r}$," Proceedings of the American Mathematical Society, vol. 54, pp. 331-336, 1976.

[12] J. Hoffmann-Jørgensen and G. Pisier, "The law of large numbers and the central limit theorem in Banach spaces," Annals of Probability, vol. 4, no. 4, pp. 587-599, 1976.

[13] S. Gan, "The Hàjek-Rènyi inequality for Banach space valued martingales and the $p$ smoothness of Banach spaces," Statistics \& Probability Letters, vol. 32, no. 3, pp. 245-248, 1997.

[14] S. Gan and D. Qiu, "On the Hájek-Rényi inequality," Wuhan University Journal of Natural Sciences, vol. 12, no. 6, pp. 971-974, 2007.

[15] T. C. Christofides and R. J. Serfling, "Maximal inequalities for multidimensionally indexed submartingale arrays," The Annals of Probability, vol. 18, no. 2, pp. 630-641, 1990.

[16] F. Móricz, U. Stadtmüller, and M. Thalmaier, "Strong laws for blockwise $\mathcal{M}$-dependent random fields," Journal of Theoretical Probability, vol. 21, no. 3, pp. 660-671, 2008. 
[17] L. V. Dung, T. Ngamkham, N. D. Tien, and A. I. Volodin, "Marcinkiewicz-Zygmund type law of large numbers for double arrays of random elements in Banach spaces," Lobachevskii Journal of Mathematics, vol. 30, no. 4, pp. 337-346, 2009.

[18] N. V. Quang and N. V. Huan, “On the strong law of large numbers and $\mathcal{L}_{p}$-convergence for double arrays of random elements in $p$-uniformly smooth Banach spaces," Statistics E Probability Letters, vol. 79, no. 18, pp. 1891-1899, 2009.

[19] L. V. Thanh, "On the strong law of large numbers for $d$-dimensional arrays of random variables," Electronic Communications in Probability, vol. 12, pp. 434-441, 2007. 